\title{
TAMANHO ÓTIMO DE AMOSTRA PARA AVALIAÇÃO DE CARACTERES DE FRUTOS DE ABACAXIZEIRO EM EXPERIMENTOS COM ADUBAÇÃO USANDO PARCELAS GRANDES ${ }^{1}$
}

\author{
WILLIAN KRAUSE ${ }^{2}$, LINDOLFO STORCK ${ }^{3}$, ALESSANDRO DAL'COL LÚCIO $^{3}, \mathrm{ASTOR}^{\circ}$ \\ HENRIQUE NIED ${ }^{4}$, REGIS QUEIROZ GONÇALVES ${ }^{4}$
}

RESUMO-O objetivo deste estudo foi determinar o tamanho ótimo de amostra para avaliar a massa, o comprimento e o diâmetro de frutos de abacaxieiro, usando parcelas grandes submetidas a diferentes adubações. No experimento com abacaxizeiro (cultivar Pérola), foram avaliadas duas fontes de nitrogênio (ureia e sulfato de amônio) e cinco doses de cloreto de potássio (zero;350; 700; 1.050 e $1.400 \mathrm{~kg} \mathrm{ha}^{-1}$ ). Cada parcela continha cinco fileiras duplas de quatro metros de comprimento, sendo que as três fileiras centrais foram consideradas como área útil. $\mathrm{O}$ espaçamento de plantio foi $1,2 \times 0,4 \times 0,4 \mathrm{~m}$, correspondente a 60 plantas por parcela útil. O delineamento experimental foi o de blocos completos casualizados, com quatro repetições. Foi calculado o tamanho de amostra $(\eta)$ para as semiamplitudes do intervalo de confiança (SA) iguais a 2; 4; 6;8 e 10\% da estimativa da média, com grau de confiança (1- $\alpha$ ) de $95 \%$, usando a distribuição $\mathrm{t}$ de Student. Posteriormente, fixou-se $\eta$ como o total de frutos colhidos por parcela para o cálculo da SA em percentagem da estimativa da média para cada um dos caracteres. Com um erro de estimação de $4 \%$ da média, devem ser amostrados, respectivamente, 83; 35 e 10 frutos em cada uma das parcelas experimentais, para a avaliação da massa, do comprimento e do diâmetro de frutos de abacaxizeiro, cultivar Pérola, em experimentos com adubação.

Termos para indexação: Ananas comosus, amostragem, experimentos com grandes unidades experimentais.

\section{OPTIMUM SAMPLE SIZE FOR FRUITS CHARACTERS OF PINEAPPLE UNDER FERTILIZATIONS EXPERIMENTS USING LARGE PLOTS}

\begin{abstract}
The aim of this study was to determine the sample size needed to evaluated the mass, length and diameter of pineapple fruits in large experimental units under different fertilizations. In the experiment with pineapple (cultivar Perola) it was evaluated two sources of nitrogen (urea and ammonium sulfate) and five doses of potassium (zero, 350, 700, 1,050 and 1,400 $\mathrm{kg} \mathrm{ha}^{-1}$ ). Each plot had five double strips of four meters length, being the three central strips taken as useful area. The plant spacing was $1,2 \times 0,4 \times 0,4 \mathrm{~m}$, making 60 plants per useful area of each plot. It was used a randomized blocks design with four replications. The sample size $(\eta)$ was calculated for the semiamplitude of the confidence interval (SA) equal to: 2, 4, 6, 8 and $10 \%$ of the average estimate, with degree of confidence (1- $\alpha$ ) of $95 \%$, using the Student's t distribution. Afterwards, $\eta$ was set as the total amount of fruits per plot for calculating SA as percentage of the average estimate for each character. With an average estimate error of $4 \%$, should be sample 83,35 and 10 fruits in each experimental plot for evaluation of mass, length and diameter, respectively, for pineapple fruits, cultivar Perola, in experiments with fertilization.
\end{abstract}

Index terms: Ananas comosus, sampling, experiments with large experimental units.

\footnotetext{
'(Trabalho 165-12). Recebido em: 17-05-2012. Aceito para publicação em: 29-11-2012. Projeto de pesquisa financiado pela FAPEMAT - Fundação de Amparo a Pesquisa do Estado de Mato Grosso.

${ }^{2}$ Doutor, Professor Adjunto, Universidade do Estado de Mato Grosso - UNEMAT. Rod. MT 358, km 07, Jardim Aeroporto, Cep 78300000, Tangará da Serra-MT. E-mail: krause@unemat.br.

${ }^{3}$ Doutores, Professores Adjuntos, Universidade Federal de Santa Maria - UFSM. Camobi, s/n, Cep 97105-900, Santa Maria-RS. E-mails: lindolfostorck@gmail.com; adlucio@ufsm.br.

${ }^{4}$ Mestres, Professores Assistentes, Universidade do Estado de Mato Grosso - UNEMAT. Rod. MT 358, km 07, Jardim Aeroporto, Cep 78300-000, Tangará da Serra-MT. E-mails: regisqg@yahoo.com.br; astornied@yahoo.com.br.
} 


\section{INTRODUÇÃO}

O abacaxizeiro, espécie Ananas comosus (L. Merril), pertence à família Bromeliácea, monocotiledônea de clima tropical, é originário do Brasil. O fruto apresenta ótimo sabor e aroma intenso, utilizado tanto para consumo in natura como para industrialização de suco, pedaços em calda e geleias. É uma das frutas cultivadas com maior exigência nutricional, apresentando alta demanda por nutriente em relação às outras espécies frutíferas (SILVA et al., 2009). O Brasil é o maior produtor mundial de abacaxi. Em 2010, foram produzidos 1.470 .391 milheiros de abacaxis, numa área de 60.016 ha, resultando em rendimento médio de 24.500 frutos ha ${ }^{-1}$.

A adubação é um fator que influencia diretamente na produtividade e na qualidade do abacaxizeiro, podendo ser limitante se o elemento químico estiver em baixa quantidade no solo ou for fornecido por meio da adubação numa quantidade insuficiente. De acordo com Paula et al. (1991), o N e o K são nutrientes requeridos em grandes quantidades.

$\mathrm{O} \mathrm{N}$, de maneira geral, aumenta o tamanho do fruto (PAULA et al., 1991) e o conteúdo do suco (VELOSO et al., 2001). Sua deficiência produz frutos coloridos e deformados, reduz o tamanho dos frutos e das coroas e inibe o crescimento vegetal. $\mathrm{O} \mathrm{K}$ influencia a qualidade, a massa e o diâmetro do fruto (PAULA et al., 1998; VELOSO et al., 2001), bem como o tamanho (PAULA et al., 1991). A deficiência de K reduz o teor de sólidos solúveis totais (PAULA et al., 1999).

Em pesquisas experimentais de campo para a determinação das necessidades nutricionais em abacaxizeiro, quando são necessárias parcelas grandes, para alguns caracteres, a avaliação de todos os frutos é muito trabalhosa. Assim, é importante obter uma amostra representativa dos frutos, devidamente dimensionada, para adequar o tempo, os recursos financeiros e os humanos disponíveis.

O tamanho de amostra é proporcional à variabilidade dos dados e à confiabilidade desejada na estimativa da média, sendo inversamente proporcional ao erro de estimação permitido a priori pelo pesquisador (BARBETTA et al., 2004). Assim, amostras de tamanho maior resultam em maior precisão experimental porque reduzem a variância da média amostral, embora a demanda por recursos também seja elevada. Por outro lado, tamanho de amostra pequeno pode reduzir a precisão experimental (FERNANDES; SILVA, 1996). O dimensionamento do tamanho ótimo de amostra melhora a eficiência da pesquisa, permitindo a obtenção de estimativas com precisão desejada (BARBETTA et al., 2004).
Vários trabalhos de determinação do tamanho de amostra para caracteres morfológicos, fenológicos e produtivos de diversas culturas agrícolas foram publicados. Pode-se citar, por exemplo, milho (FERNANDES; SILVA, 1996; SILVA et al., 1998; STORCK et al., 2007), milho-pipoca (CATAPATTI et al., 2008), cana-de-açúcar (LEITE et al., 2009), soja (CARGNELUTTI FILHO et al., 2009), crambe (CARGNELUTTI FILHO et al., 2010b), mamoneira (CARGNELUTTI FILHO et al., 2010a) e feijão-de-vagem (HAESBAERT et al., 2011). Ainda, outros trabalhos foram conduzidos para determinar o tamanho de amostra na quantificação de lesões de doenças, como o cancro-cítrico (BELASQUE JÚNIOR et al., 2008) e na quantificação de pragas, como o ácaro-do-bronzeado (LÚCIO et al., 2009). Contudo, não foram encontrados na literatura trabalhos que determinam o tamanho ótimo de amostra para avaliar a massa, o comprimento e o diâmetro de frutos em experimentos de abacaxizeiro. Também, não foram encontradas publicações referentes ao tamanho ótimo de parcela específico para esta cultura. No entanto, na literatura, são relatados usos de parcelas de tamanhos bem variados: cinco plantas (COELHO et al., 2007), 12 plantas úteis (SOUZA et al., 2009) e 32 plantas úteis (VELOSO et al., 2001). Assim, não há informação específica sobre o tamanho de amostra nem sobre o tamanho de parcela e número de repetições para a avaliação de frutos do abacaxizeiro.

Desta forma, o objetivo deste estudo foi determinar o tamanho ótimo de amostra para avaliar a massa, o comprimento e o diâmetro de frutos de abacaxizeiro em ensaios de adubação, usando parcelas grandes, submetidos a diferentes adubações.

\section{MATERIAL E MÉTODOS}

O experimento com abacaxizeiro (cultivar Pérola) foi conduzido na área experimental $\left(14^{\circ} 39^{\prime}\right.$ sul, $57^{\circ} 25^{\prime}$ oeste, $320 \mathrm{~m}$ de altitude) do Câmpus Universitário de Tangará da Serra-MT (UNEMAT). O solo é classificado como Latossolo Vermelho distroférrico, de textura argilosa e de relevo plano a levemente ondulado. O clima da região é caracterizado por precipitação média anual de $1.800 \mathrm{~mm}$ e temperatura média anual de $24,4{ }^{\circ} \mathrm{C}$.

Foram avaliadas duas formulações de nitrogênio $\left(1.333 \mathrm{~kg}\right.$ de ureia $\mathrm{ha}^{-1}$ e $3.000 \mathrm{~kg}$ de sulfato de amônio ha-1) e cinco doses de cloreto de potássio (zero; 350; 700; 1.050 e $1.400 \mathrm{~kg} \mathrm{ha}^{-1}$ ). Cada parcela continha cinco fileiras duplas de quatro metros de comprimento, sendo que as três fileiras centrais foram consideradas como área útil. $\mathrm{O}$ espaçamento de plantio foi $1,2 \times 0,4 \times 0,4 \mathrm{~m}$, correspondente a 60 
plantas por parcela útil. O delineamento experimental foi o de blocos completos casualizados, com quatro repetições.

O solo foi preparado de forma convencional, com adubação de acordo com as análises e as recomendações (CUNHA et al., 1999). Foram aplicados no sulco 9,0 g MAP linha ${ }^{-1}$ e ambas as fontes de nitrogênio. As doses de cloreto de potássio foram divididas em três aplicações, sendo aos 90; 120 e 160 dias após o plantio (SPIRONELLO et al., 2004).

As mudas foram selecionadas e separadas de acordo com o tipo e tamanho, sendo que, durante a seleção, foram descartadas as que se apresentavam defeituosas ou com sintomas de doenças. O plantio das mudas selecionadas foi realizado manualmente, no dia 10 de novembro de 2009. A partir do plantio, a umidade do solo foi mantida com irrigação por aspersão. A indução floral foi realizada 250 dias após o plantio, com a aplicação de Ethrel (ethephon) (50 $\mathrm{ml}$ planta $\left.^{-1}\right)$, na forma líquida, colocado no centro da roseta foliar. Durante o experimento, foram realizadas as práticas culturais usuais, visando a garantir as condições de limpeza e de sanidade das plantas.

A colheita foi realizada nos meados de dezembro de 2010 (120 dias após a indução floral). Foi determinada a massa dos frutos $\left(\mathrm{g}\right.$ fruto $^{-1}$, sem a coroa) aptos ao consumo. Dos frutos colhidos, foi obtida uma amostra aleatória de dez frutos de cada parcela para a determinação do comprimento (mm, sem a coroa) e diâmetro ( $\mathrm{mm}$, na parte média) dos frutos.

Para cada característica mensurada nos frutos (massa, comprimento e diâmetro) obtidos de cada uma das parcelas, foi procedida uma análise da variância, segundo o modelo do delineamento em blocos ao acaso, com quatro repetições e $\mathrm{k}$ amostras por parcela. Foram determinadas as estatísticas descritivas (média, mínimo, máximo, desvio-padrão e coeficiente de variação). Para verificar a possibilidade do uso da distribuição $t$ de Student na estimação do tamanho ótimo de amostra, foram testadas as hipóteses referentes à assimetria (Ho: assimetria $=0$, pelo teste $t$ com $p=0,05)$, à curtose $($ Ho: curtose $=3$, pelo teste $t$ com $p=0,05)$ e aderência à distribuição normal, pelo teste de Lilliefors $(\mathrm{p}=0,05)$ (BARBETTA et al., 2004). Para estes testes, foi usado o aplicativo computacional SAEG (2007).

Foi calculado o tamanho de amostra $(\eta)$ para as semiamplitudes do intervalo de confiança (SA) iguais a $2 ; 4 ; 6 ; 8$ e $10 \%$ da estimativa da média (m), com grau de confiança $(1-\alpha)$ de $95 \%$, por meio da expressão $\eta=\underline{t_{\alpha / 2}^{2} s^{2}}$

$$
\eta=\frac{t_{\alpha / 2}^{2} s^{2}}{(S A)^{2}} \text { (BARBETTA et al., 2004)' }
$$

sendo $t_{\alpha / 2} o$ valor crítico da distribuição $t$ de Student, tal que $\mathrm{P}\left(\mathrm{t}>\mathrm{t}_{\alpha / 2}\right)=\alpha / 2$, com (n-1) graus de liberdade; com $\alpha=5 \%$ de probabilidade de erro, e $\mathrm{s}^{2}$ é a estimativa de variância. Posteriormente, fixou-se $\eta$ como o total de frutos colhidos por parcela para o cálculo da SA, em percentagem da estimativa da média $(\mathrm{m})$ para cada um dos caracteres, por meio da expressão

$$
\mathrm{SA}=100 \frac{\mathrm{t}_{\alpha / 2}{ }^{\mathrm{s}}}{\mathrm{m} \sqrt{\eta}} \quad \text { (BARBETTA et al., }
$$

2004), em que s é a estimativa do desvio- padrão amostral. Para os cálculos, foram usados a planilha Excel e o aplicativo SAEG (2007).

\section{RESULTADOS E DISCUSSÃO}

Para os caracteres massa, comprimento e diâmetro, as estimativas dos componentes de variação entre parcelas $\left(\hat{\sigma}^{2}\right)$ foram menores do que as estimativas dos componentes de variação dentro de parcela $\left(\hat{\sigma}_{\varepsilon}^{2}\right)$, sendo que, para o caráter massa e diâmetro de fruto, a variância entre parcela é significativamente ( $\mathrm{p}$ $<0,05$ ) maior do que zero (Tabela 1 ). Neste caso, um plano amostral que usa um mesmo número de frutos por tratamento deve proporcionar maior tamanho de amostra em detrimento do número de repetições, para a comparação de médias de tratamentos com maior precisão.

Para os caracteres comprimento e diâmetro de frutos, o coeficiente de variação, entre e dentro de parcela, foi classificado como baixo (próximo a 10\%), conforme critério usual, para uso em experimentos agrícolas, em que o abacaxizeiro poderia estar incluído. Usando o mesmo critério, para o caráter massa de frutos, o coeficiente de variação entre parcelas $(49,8 \%)$ foi muito alto e dentro de parcelas $(15,6 \%)$ foi classificado como médio. Assim, para a estimação da média com mesma precisão, o tamanho de amostra para características de comprimento e diâmetro pode ser menor em relação à massa de fruto. Para o caráter massa de fruto, os coeficientes de variação obtidos dentro de cada parcela (Tabela 2 ) foram classificados na mesma faixa de precisão média. Ao verificar os valores mínimos e máximos, pode-se ter uma ideia da amplitude de variação (medida de variabilidade) dos valores que justificam as magnitudes dos respectivos desvio-padrão e coeficiente de variação. Da mesma forma, para os caracteres comprimento e diâmetro de fruto, os coeficientes de variação obtidos dentro das quatro parcelas de cada tratamento (Tabela 3) também se classificam na mesma faixa de precisão alta (coeficiente de variação baixo).

Para os três caracteres (massa, comprimento e diâmetro), as hipóteses da assimetria igual a zero, 
a curtose diferente de três e o ajuste à distribuição normal não foram rejeitados a $5 \%$ de probabilidade. A exceção foi para uma das parcelas referentes ao caráter massa de fruto, em que a curtose foi diferente de três, mesmo assim este fato não interferiu no ajuste à distribuição normal deste caráter. $\mathrm{O}$ fato de os dados obtidos atenderem aos pressupostos (assimetria, curtose e normalidade) confere a adequabilidade dos mesmos para a estimação do tamanho de amostra com base na distribuição t de Student, sem a necessidade de transformação dos dados ou o uso de outros métodos estatísticos, como a reamostragem bootstrap.

O tamanho de amostra (número de frutos), para a estimação da média da massa (média de quatro repetições), com semiamplitude do intervalo de confiança igual a $2 \%$ da estimativa da média $(\mathrm{SA}=2 \%)$, foi de 421 frutos para adubação com ureia e dose nula de cloreto de potássio, de 249 frutos para adubação com sulfato de amônio e $350 \mathrm{~kg} \mathrm{ha}^{-1}$ de cloreto de potássio (Tabela 4), confirmando a variabilidade relacionada à forma de adubação. No entanto, em níveis de precisão menores (maior SA) as diferenças no tamanho de amostra, relacionadas às formas de adubação, são bem menos aparentes. Na média (duas formas de adubação e cinco doses), o tamanho de amostra, para $\mathrm{SA}=2 \%$, é igual a $(324+339) / 2=331$ frutos para a avaliação da massa de frutos por parcela.

Um resultado importante que pode ser observado é a homogeneidade do tamanho de amostra, entre parcelas de cada tratamento e entre médias de tratamentos. Esta situação é particularmente importante porque irá facilitar a execução do experimento, na parte de coleta dos resultados (mesmo tamanho de amostra para todas as parcelas) e também por atender aos pressupostos para os testes de hipóteses. As mesmas considerações são válidas para os caracteres comprimento e diâmetro de frutos, em magnitudes inferiores.

Embora com o número elevado de frutos (média de 331 frutos) a serem avaliados para a determinação da massa de frutos, com esse nível de confiança $(\mathrm{SA}=2 \%)$, em outras culturas agrícolas, é comum obter-se tamanhos de amostra com essa magnitude. Neste sentido, alguns estudos em caracteres morfológicos e produtivos da cultura de crambe (CARGNELUTTI FILHO et al., 2010b) mostram que, nesse nível de confiança $(\mathrm{SA}=2 \%)$, é necessário avaliar de 379 a 3.811 plantas. Para estimar o diâmetro das lesões de cancro- cítrico, Belasque Júnior et al. (2008) indicam a mensuração de 148 a 307 lesões para $\mathrm{SA}=2 \%$. Já em mamoneira, Cargnelutti Filho et al. (2010a) observaram que, para $\mathrm{SA}=2 \%$, em alguns caracteres, é necessário avaliar
6.077 plantas. Estes tamanhos de amostra elevados geralmente inviabilizam a coleta de informações neste nível de confiança ( $\mathrm{SA}=2 \%)$. Nestes casos, níveis de precisão menores (maior SA) devem ser considerados suficientes devido à impossibilidade prática de usar tamanhos de amostra tão elevados. Alem disso, é possível que o tamanho de amostra estimado extrapole o próprio número de observações disponíveis nas parcelas.

Como foram calculados os tamanhos de amostra com diferentes erros de estimação ( $\mathrm{SA}=2 ; 4$; 6; 8 e 10\%), o leitor tem a possibilidade da escolha da taxa de erro mais adequado para o seu caso. Para a massa de fruto, 14 frutos por parcela (média dos 10 tratamentos) são suficientes para a estimação da média, com uma SA=10\% (Tabela 4). Nesta mesma condição, são necessários apenas cinco frutos para avaliar o comprimento e dois frutos para avaliar o diâmetro. A ocorrência de variabilidade do tamanho de amostra para diferentes caracteres mensuradas já foi relatada para as culturas de milho (STORCK et al., 2007), de soja (CARGNELUTTI FILHO et al., 2009), de mamona (CARGNELUTTI FILHO et al., 2010a) e de crambe (CARGNELUTTI FILHO et al., 2010b). No entanto, considerando a dificuldade em amostrar diferentes quantidades de frutos para cada caráter, a média geral, em princípio, pode ser adequada. Neste sentido, considerando $\mathrm{SA}=4 \%$ da média, o tamanho ótimo de amostra é igual a 83;35 e 10 frutos por parcela, respectivamente, para a avaliação da massa, do comprimento e do diâmetro de frutos de abacaxizeiro (Tabela 4, média geral). 
TABELA 1- Análise de variância com graus de liberdade (GL) e quadrado médio (QM) das fontes de variação (FV), blocos, tratamentos (fontes de nitrogênio e doses de cloreto de potássio), variação entre (VE) e dentro (VD) de parcelas, média, coeficiente de variação (CV), estimativa do componente de variação entre $\left(\sigma^{2}\right)$ e dentro $\left(\sigma_{\varepsilon}^{2}\right)$ de parcela, para diferentes caracteres de frutos de abacaxizeiro.

\begin{tabular}{lcccccc}
\hline \multirow{2}{*}{ FV } & \multicolumn{2}{c}{ Massa $\left(\mathrm{g}\right.$ fruto $\left.^{-1}\right)$} & \multicolumn{2}{c}{ Comprimento $(\mathrm{mm})$} & \multicolumn{2}{c}{ Diâmetro $(\mathrm{mm})$} \\
\cline { 2 - 7 } & GL & QM & GL & QM & GL & QM \\
\hline Bloco & 3 & $2595114,2^{*}$ & 3 & $2230,5^{*}$ & 3 & $304,9^{*}$ \\
Tratamento & 9 & $658985,9^{\text {ns }}$ & 9 & $467,1^{\text {ns }}$ & 9 & $103,7^{\text {ns }}$ \\
VE & 27 & $299456,9^{*}$ & 27 & $231,2^{\text {ns }}$ & 27 & $56,9^{*}$ \\
VD & 2005 & 29524,7 & 360 & 156,3 & 360 & 25,8 \\
Média & - & 1099,4 & - & 141,5 & - & 107,6 \\
CV(VE)\% & - & 49,8 & - & 10,7 & - & 7,0 \\
CV(VD)\% & - & 15,6 & - & 8,8 & - & 4,7 \\
& & 5292,8 & & 7,5 & & 3,1 \\
& & 29524,7 & & 156,3 & & 25,8 \\
\hline
\end{tabular}

* Efeito significativo pelo teste $\mathrm{F}(\mathrm{p}<5 \%)$; ${ }^{\text {ns }}$ efeito não significativo pelo teste $\mathrm{F}$.

TABELA 2 - Número de frutos mensurados (N), valor mínimo (Mín), valor máximo (Máx), média, desvio-padrão (DP), coeficiente de variação (CV\%), estimativa da assimetria (AS) e da curtose+3 (CT) e resultado do teste de normalidade de Lilliefors (TL) para a massa de frutos ( $\mathrm{g}$ fruto ${ }^{-1}$ ) de abacaxizeiro avaliados em parcelas sob diferentes fontes de nitrogênico (FN) e doses de cloreto de potássio (D, $\left.\mathrm{kg} \mathrm{ha}^{-1}\right)$ em quatro repetições (Rep).

\begin{tabular}{|c|c|c|c|c|c|c|c|c|c|c|c|}
\hline $\mathrm{FN}^{(4)}$ & D & Rep & $\mathrm{N}$ & Mín & Máx & Média & DP & $\mathrm{CV}(\%)$ & $\mathrm{AS}^{(1)}$ & $\mathrm{CT}^{(2)}$ & $\mathrm{TL}^{(3)}$ \\
\hline SA & 0 & 1 & 47 & 685 & 1290 & 939 & 150,5 & 16,0 & $0,44 \mathrm{~ns}$ & $2,46 \mathrm{~ns}$ & ns \\
\hline SA & 0 & 2 & 37 & 800 & 1990 & 1279 & 265,1 & 20,7 & $0,36 \mathrm{~ns}$ & $2,66 \mathrm{~ns}$ & ns \\
\hline SA & 0 & 3 & 48 & 290 & 1505 & 1167 & 198,3 & 17,0 & $-1,62 \mathrm{~ns}$ & $8,77^{*}$ & ns \\
\hline SA & 0 & 4 & 46 & 805 & 1745 & 1179 & 172,3 & 14,6 & $0,65 \mathrm{~ns}$ & $3,84 \mathrm{~ns}$ & ns \\
\hline SA & 350 & 1 & 54 & 700 & 1280 & 1001 & 128,6 & 12,8 & $-0,13 \mathrm{~ns}$ & $2,23 \mathrm{~ns}$ & ns \\
\hline SA & 350 & 2 & 60 & 840 & 1730 & 1194 & 178,4 & 14,9 & $0,61 \mathrm{~ns}$ & $3,32 \mathrm{~ns}$ & ns \\
\hline SA & 350 & 3 & 58 & 945 & 1680 & 1286 & 186,3 & 14,4 & $0,25 \mathrm{~ns}$ & $2,11 \mathrm{~ns}$ & ns \\
\hline SA & 350 & 4 & 40 & 900 & 1535 & 1219 & 149,0 & 12,2 & $-0,02 \mathrm{~ns}$ & $2,43 \mathrm{~ns}$ & ns \\
\hline SA & 700 & 1 & 47 & 720 & 1345 & 1035 & 142,8 & 13,8 & $-0,22 \mathrm{~ns}$ & $2,57 \mathrm{~ns}$ & ns \\
\hline SA & 700 & 2 & 54 & 920 & 1715 & 1235 & 165,4 & 13,4 & $0,65 \mathrm{~ns}$ & $3,12 \mathrm{~ns}$ & ns \\
\hline SA & 700 & 3 & 49 & 980 & 1455 & 1216 & 115,6 & 9,5 & $-0,05 \mathrm{~ns}$ & $2,20 \mathrm{~ns}$ & ns \\
\hline SA & 700 & 4 & 43 & 535 & 1370 & 1027 & 206,4 & 20,1 & $0,05 \mathrm{~ns}$ & $2,13 \mathrm{~ns}$ & ns \\
\hline SA & 1050 & 1 & 49 & 600 & 1295 & 1012 & 154,2 & 15,2 & $-0,49 \mathrm{~ns}$ & $2,47 \mathrm{~ns}$ & ns \\
\hline SA & 1050 & 2 & 46 & 970 & 1635 & 1288 & 158,1 & 12,3 & $0,19 \mathrm{~ns}$ & $2,22 \mathrm{~ns}$ & ns \\
\hline SA & 1050 & 3 & 56 & 645 & 1525 & 1014 & 183,2 & 18,1 & $0,38 \mathrm{~ns}$ & $2,93 \mathrm{~ns}$ & ns \\
\hline SA & 1050 & 4 & 51 & 765 & 1755 & 1164 & 219,9 & 18,9 & $0,43 \mathrm{~ns}$ & $2,81 \mathrm{~ns}$ & ns \\
\hline SA & 1400 & 1 & 53 & 690 & 1340 & 1020 & 151,1 & 14,8 & $-0,06 \mathrm{~ns}$ & $2,06 \mathrm{~ns}$ & ns \\
\hline SA & 1400 & 2 & 55 & 920 & 1580 & 1238 & 147,6 & 11,9 & $0,14 \mathrm{~ns}$ & $2,61 \mathrm{~ns}$ & ns \\
\hline SA & 1400 & 3 & 53 & 815 & 1820 & 1208 & 197,3 & 16,3 & $0,84 \mathrm{~ns}$ & $4,12 \mathrm{~ns}$ & ns \\
\hline SA & 1400 & 4 & 54 & 585 & 1490 & 1050 & 194,1 & 18,5 & $-0,11 \mathrm{~ns}$ & $2,75 \mathrm{~ns}$ & ns \\
\hline $\mathrm{U}$ & 0 & 1 & 47 & 620 & 1350 & 971 & 176,2 & 18,1 & $0,13 \mathrm{~ns}$ & $2,34 \mathrm{~ns}$ & ns \\
\hline $\mathrm{U}$ & 0 & 2 & 52 & 450 & 1435 & 1043 & 187,5 & 17,9 & $-0,16 \mathrm{~ns}$ & $3,67 \mathrm{~ns}$ & ns \\
\hline $\mathrm{U}$ & 0 & 3 & 52 & 485 & 1560 & 1044 & 180,7 & 17,3 & $0,22 \mathrm{~ns}$ & $4,68 \mathrm{~ns}$ & ns \\
\hline $\mathrm{U}$ & 0 & 4 & 35 & 700 & 1515 & 1114 & 192,7 & 17,3 & $0,26 \mathrm{~ns}$ & $2,63 \mathrm{~ns}$ & ns \\
\hline $\mathrm{U}$ & 350 & 1 & 48 & 740 & 1545 & 1090 & 141,1 & 12,9 & $0,52 \mathrm{~ns}$ & $4,15 \mathrm{~ns}$ & ns \\
\hline $\mathrm{U}$ & 350 & 2 & 52 & 750 & 1500 & 1051 & 175,1 & 16,6 & $0,34 \mathrm{~ns}$ & $2,40 \mathrm{~ns}$ & ns \\
\hline $\mathrm{U}$ & 350 & 3 & 53 & 845 & 1730 & 1212 & 170,3 & 14,0 & $0,56 \mathrm{~ns}$ & $3,77 \mathrm{~ns}$ & ns \\
\hline U & 350 & 4 & 52 & 675 & 1545 & 1104 & 173,9 & 15,7 & $0,30 \mathrm{~ns}$ & $3,12 \mathrm{~ns}$ & ns \\
\hline U & 700 & 1 & 60 & 825 & 1395 & 1050 & 143,8 & 13,7 & $0,53 \mathrm{~ns}$ & $2,73 \mathrm{~ns}$ & ns \\
\hline $\mathrm{U}$ & 700 & 2 & 53 & 885 & 1730 & 1223 & 173,9 & 14,2 & $0,25 \mathrm{~ns}$ & $3,10 \mathrm{~ns}$ & ns \\
\hline $\mathrm{U}$ & 700 & 3 & 51 & 570 & 1425 & 1058 & 183,7 & 17,4 & $-0,48 \mathrm{~ns}$ & $2,92 \mathrm{~ns}$ & ns \\
\hline $\mathrm{U}$ & 700 & 4 & 51 & 580 & 1525 & 1062 & 197,9 & 18,6 & $0,12 \mathrm{~ns}$ & $2,59 \mathrm{~ns}$ & ns \\
\hline U & 1050 & 1 & 58 & 700 & 1340 & 1001 & 142,1 & 14,2 & $0,10 \mathrm{~ns}$ & $2,55 \mathrm{~ns}$ & ns \\
\hline $\mathrm{U}$ & 1050 & 2 & 55 & 415 & 1500 & 1101 & 189,9 & 17,2 & $-0,63 \mathrm{~ns}$ & $4,71 \mathrm{~ns}$ & ns \\
\hline U & 1050 & 3 & 56 & 680 & 1555 & 1197 & 195,6 & 16,3 & $-0,04 n s$ & $2,47 \mathrm{~ns}$ & ns \\
\hline $\mathrm{U}$ & 1050 & 4 & 52 & 585 & 1310 & 1022 & 152,4 & 14,9 & $-0,42 \mathrm{~ns}$ & $3,44 \mathrm{~ns}$ & ns \\
\hline $\mathrm{U}$ & 1400 & 1 & 55 & 610 & 1200 & 919 & 129,8 & 14,1 & $-0,08 \mathrm{~ns}$ & $2,41 \mathrm{~ns}$ & ns \\
\hline $\mathrm{U}$ & 1400 & 2 & 56 & 755 & 1200 & 984 & 111,3 & 11,3 & $-0,09 \mathrm{~ns}$ & $2,44 \mathrm{~ns}$ & ns \\
\hline $\mathrm{U}$ & 1400 & 3 & 54 & 490 & 1345 & 1016 & 194,0 & 19,1 & $-0,57 \mathrm{~ns}$ & $3,17 \mathrm{~ns}$ & ns \\
\hline $\mathrm{U}$ & 1400 & 4 & 53 & 710 & 1330 & 992 & 147,6 & 14,9 & $0,16 \mathrm{~ns}$ & $2,22 \mathrm{~ns}$ & ns \\
\hline
\end{tabular}

(1) *Assimetria difere de zero, pelo teste $\mathrm{t}(\mathrm{p}=0,05)^{\mathrm{ns}}=$ não significativo; ${ }^{(2)} *$ Curtose difere de três, pelo teste $\mathrm{t}(\mathrm{p}=0,05) ;{ }^{(3)} \mathrm{ns}$ Distribuição normal $(\mathrm{p}=0,05) ;{ }^{(4)} \mathrm{SA}=$ Sulfato de amônio $\left(3.000 \mathrm{~kg} \mathrm{ha}^{-1}\right), \mathrm{U}=$ Ureia $\left(1.333 \mathrm{~kg} \mathrm{ha}^{-1}\right)$. 
TABELA 3 - Número de frutos mensurados (N), valor mínimo (Mín), valor máximo (Máx), média, desviopadrão (DP), coeficiente de variação (CV\%), estimativa da assimetria (AS) e da curtose+3 (CT) e resultado do teste de normalidade de Lilliefors (TL) para o comprimento e diâmetro de frutos $(\mathrm{mm})$ de abacaxizeiro, na média de quatro parcelas, sob diferentes fontes de nitrogênico (FN) e doses de cloreto de potássio (D, $\left.\mathrm{kg} \mathrm{ha}^{-1}\right)$.

\begin{tabular}{|c|c|c|c|c|c|c|c|c|c|c|}
\hline $\mathrm{FN}^{(4)}$ & $\mathrm{D}$ & $\mathrm{N}$ & Mín & Máx & Média & $\mathrm{DP}$ & $\mathrm{CV}(\%)$ & $\mathrm{AS}^{(1)}$ & $\mathrm{CT}^{(2)}$ & $\mathrm{TL}^{(3)}$ \\
\hline & \multicolumn{10}{|c|}{ Comprimento de fruto $(\mathrm{mm})$} \\
\hline SA & 0 & 40 & 105,8 & 164,8 & 142,6 & 14,7 & 10,3 & $-0,57 \mathrm{~ns}$ & $2,20 \mathrm{~ns}$ & ns \\
\hline SA & 350 & 40 & 108,8 & 177,9 & 146,5 & 15,2 & 10,3 & $-0,15 \mathrm{~ns}$ & $3,02 \mathrm{~ns}$ & ns \\
\hline SA & 700 & 40 & 103,7 & 160,3 & 140,4 & 12,7 & 9,0 & $-0,51 \mathrm{~ns}$ & $3,00 \mathrm{~ns}$ & ns \\
\hline SA & 1050 & 40 & 103,6 & 192,0 & 142,6 & 17,4 & 12,2 & $0,24 \mathrm{~ns}$ & $3,45 \mathrm{~ns}$ & ns \\
\hline SA & 1400 & 40 & 125,3 & 160,4 & 145,0 & 10,6 & 7,3 & $-0,48 \mathrm{~ns}$ & $1,84 \mathrm{~ns}$ & ns \\
\hline $\mathrm{U}$ & 0 & 40 & 110,4 & 166,4 & 136,7 & 12,0 & 8,8 & $0,23 \mathrm{~ns}$ & $2,77 \mathrm{~ns}$ & ns \\
\hline $\mathrm{U}$ & 350 & 40 & 115,8 & 170,5 & 143,1 & 12,6 & 8,8 & $-0,18 \mathrm{~ns}$ & $2,97 \mathrm{~ns}$ & ns \\
\hline $\mathrm{U}$ & 700 & 40 & 118,3 & 186,3 & 142,8 & 13,5 & 9,4 & $0,58 \mathrm{~ns}$ & $3,72 \mathrm{~ns}$ & ns \\
\hline $\mathrm{U}$ & 1050 & 40 & 106,8 & 160,4 & 139,4 & 12,6 & 9,0 & $-0,46 \mathrm{~ns}$ & $2,63 \mathrm{~ns}$ & ns \\
\hline $\mathrm{U}$ & 1400 & 40 & 112,5 & 156,4 & 135,8 & 10,3 & 7,5 & $-0,16 \mathrm{~ns}$ & $2,50 \mathrm{~ns}$ & ns \\
\hline \multicolumn{11}{|c|}{ Diâmetro de fruto (mm) } \\
\hline SA & 0 & 40 & 96,6 & 118,9 & 109,4 & 5,4 & 4,9 & $-0,19 \mathrm{~ns}$ & $2,26 \mathrm{~ns}$ & ns \\
\hline SA & 350 & 40 & 95,5 & 118,2 & 108,9 & 6,0 & 5,5 & $-0,21 \mathrm{~ns}$ & $1,18 \mathrm{~ns}$ & ns \\
\hline SA & 700 & 40 & 97,3 & 121,4 & 108,8 & 5,2 & 4,7 & $0,14 \mathrm{~ns}$ & $2,77 \mathrm{~ns}$ & ns \\
\hline SA & 1050 & 40 & 92,2 & 120,2 & 109,2 & 7,1 & 6,5 & $-0,56 \mathrm{~ns}$ & $2,34 \mathrm{~ns}$ & ns \\
\hline SA & 1400 & 40 & 97,6 & 120,0 & 107,4 & 5,4 & 5,0 & $0,23 \mathrm{~ns}$ & $2,32 \mathrm{~ns}$ & ns \\
\hline U & 0 & 40 & 96,9 & 115,8 & 107,2 & 4,6 & 4,3 & $-0,13 \mathrm{~ns}$ & $1,95 \mathrm{~ns}$ & ns \\
\hline $\mathrm{U}$ & 350 & 40 & 96,5 & 117,4 & 107,7 & 4,4 & 4,1 & $0,17 \mathrm{~ns}$ & $2,91 \mathrm{~ns}$ & ns \\
\hline U & 700 & 40 & 94,4 & 118,0 & 107,7 & 5,7 & 5,3 & $-0,48 \mathrm{~ns}$ & $3,04 \mathrm{~ns}$ & ns \\
\hline U & 1050 & 40 & 93,9 & 119,8 & 106,1 & 5,7 & 5,4 & $-0,12 \mathrm{~ns}$ & $2,80 \mathrm{~ns}$ & ns \\
\hline U & 1400 & 40 & 92,7 & 115,8 & 104,2 & 4,9 & 4,7 & $-0,17 \mathrm{~ns}$ & $2,91 \mathrm{~ns}$ & ns \\
\hline
\end{tabular}

(1) * Assimetria difere de zero, pelo teste $\mathrm{t}(\mathrm{p}=0,05),{ }^{\mathrm{ns}}=$ não significativo; ${ }^{(2)} *$ Curtose difere de três, pelo teste $\mathrm{t}(\mathrm{p}=0,05)$; ${ }^{(3)} \mathrm{ns}$ Distribuição normal $(\mathrm{p}=0,05) ;{ }^{(4)} \mathrm{SA}=$ Sulfato de amônio $\left(3.000 \mathrm{~kg} \mathrm{ha}^{-1}\right), \mathrm{U}=$ Ureia $\left(1.333 \mathrm{~kg} \mathrm{ha}^{-1}\right)$.

TABELA 4 - Tamanho de amostra (número de frutos, média de quatro repetições) para a estimação da média do comprimento, do diâmetro e da massa de frutos de abacaxizeiro, avaliados sob diferentes fontes de nitrogênico (Sulfato de amônio e Ureia) e doses de cloreto de potássio $\left(\mathrm{kg} \mathrm{ha}^{-1}\right)$, para os erros de estimação iguais a $\mathrm{SA}=2 ; 4 ; 6 ; 8$ e $10 \%$ da estimativa da média e semiamplitude do intervalo de confiança (SAI\%), com base no número de frutos mensurados $(\mathrm{N}$, média das quatro repetições).

\begin{tabular}{|c|c|c|c|c|c|c|c|c|}
\hline FN & Dose & $\mathrm{N}$ & $\mathrm{SA}=2 \%$ & $\mathrm{SA}=4 \%$ & $\mathrm{SA}=6 \%$ & $\mathrm{SA}=8 \%$ & $\mathrm{SA}=10 \%$ & $\mathrm{SAI} \%$ \\
\hline \multicolumn{9}{|c|}{ Comprimento de fruto } \\
\hline Sulf Amônio & 0 & 10 & 185 & 46 & 21 & 12 & 7 & 8,3 \\
\hline Sulf Amônio & 350 & 10 & 140 & 35 & 16 & 9 & 6 & 7,3 \\
\hline Sulf Amônio & 700 & 10 & 124 & 31 & 14 & 8 & 5 & 6,9 \\
\hline Sulf Amônio & 1050 & 10 & 232 & 58 & 26 & 14 & 9 & 9,6 \\
\hline Sulf Amônio & 1400 & 10 & 98 & 25 & 11 & 6 & 4 & 6,2 \\
\hline Média & & - & 156 & 39 & 17 & 10 & 6 & 7.7 \\
\hline Ureia & 0 & 10 & 129 & 32 & 14 & 8 & 5 & 7,1 \\
\hline Ureia & 350 & 10 & 140 & 35 & 16 & 9 & 6 & 7,4 \\
\hline Ureia & 700 & 10 & 161 & 40 & 18 & 10 & 6 & 7,9 \\
\hline Ureia & 1050 & 10 & 128 & 32 & 14 & 8 & 5 & 7,1 \\
\hline Ureia & 1400 & 10 & 76 & 19 & 8 & 5 & 3 & 5,5 \\
\hline Média & & - & 127 & 32 & 14 & 8 & 5 & 7,0 \\
\hline Média geral & & - & 141 & 35 & 16 & 9 & 6 & 7,3 \\
\hline \multicolumn{9}{|c|}{ Diâmetro de fruto } \\
\hline Sulf Amônio & 0 & 10 & 36 & 9 & 4 & 2 & 1 & 3,6 \\
\hline Sulf Amônio & 350 & 10 & 45 & 11 & 5 & 3 & 2 & 4,2 \\
\hline Sulf Amônio & 700 & 10 & 37 & 9 & 4 & 2 & 1 & 3,8 \\
\hline
\end{tabular}




\begin{tabular}{ccccccccc} 
Sulf Amônio & 1050 & 10 & 52 & 13 & 6 & 3 & 2 & 4,4 \\
Sulf Amônio & 1400 & 10 & 45 & 11 & 5 & 3 & 2 & 4,2 \\
Média & & - & 43 & 11 & 5 & 3 & 2 & 4,0 \\
Ureia & 0 & 10 & 34 & 8 & 4 & 2 & 1 & 3,7 \\
Ureia & 350 & 10 & 25 & 6 & 3 & 2 & 1 & 3,1 \\
Ureia & 700 & 10 & 52 & 13 & 6 & 3 & 2 & 4,5 \\
Ureia & 1050 & 10 & 46 & 11 & 5 & 3 & 2 & 4,2 \\
Ureia & 1400 & 10 & 32 & 8 & 4 & 2 & 1 & 3,6 \\
Média & & - & 38 & 9 & 4 & 2 & 2 & 3,8 \\
Média geral & & - & 40 & 10 & 5 & 3 & 2 & 3,9 \\
\hline \multicolumn{6}{c}{} & & \multicolumn{6}{c}{ Massa de fruto } & & & & \\
Sulf Amônio & 0 & 45 & 402 & 100 & 45 & 25 & 16 & 6,0 \\
Sulf Amônio & 350 & 53 & 249 & 62 & 28 & 16 & 10 & 4,3 \\
Sulf Amônio & 700 & 48 & 290 & 73 & 32 & 18 & 12 & 4,8 \\
Sulf Amônio & 1050 & 51 & 356 & 89 & 40 & 22 & 14 & 5,2 \\
Sulf Amônio & 1400 & 54 & 323 & 81 & 36 & 20 & 13 & 4,8 \\
Média & & - & 324 & 81 & 36 & 20 & 13 & 5,0 \\
Ureia & 0 & 47 & 421 & 105 & 47 & 26 & 17 & 6,1 \\
Ureia & 350 & 51 & 297 & 74 & 33 & 19 & 12 & 4,8 \\
Ureia & 700 & 54 & 346 & 86 & 38 & 22 & 14 & 5,1 \\
Ureia & 1050 & 55 & 328 & 82 & 36 & 21 & 13 & 4,8 \\
Ureia & 1400 & 55 & 304 & 76 & 34 & 19 & 12 & 4,6 \\
Média & & - & 339 & 85 & 38 & 21 & 14 & 5,1 \\
Média geral & & - & 331 & 83 & 37 & 21 & 13 & 5,1 \\
\hline
\end{tabular}

\section{CONCLUSÃO}

Com um erro de estimação de $4 \%$ da média, devem ser amostrados, respectivamente, $83 ; 35$ e 10 frutos em cada uma das parcelas experimentais, para a avaliação da massa, do comprimento e do diâmetro de frutos de abacaxizeiro, cultivar Pérola, em experimentos de adubação com parcelas grandes.

\section{AGRADECIMENTOS}

À FAPEMAT - Fundação de Amparo à Pesquisa do Estado de Mato Grosso, pelo financiamento do projeto de pesquisa.

\section{REFERÊNCIAS}

BARBETTA, P. A.; REIS, M.M.; BORNIA, A.C. Estatística para cursos de engenharia e informática. São Paulo: Atlas, 2004. 410p.

BELASQUE JÚNIOR, J.; JACIANI, F.J.; MARIN, D.R.; BARBOSA, J.C. Tamanho da amostra para quantificação do diâmetro de lesões de cancro cítrico. Tropical Plant Pathology, Viçosa, v.33, n.4, p.317-322, 2008.
CARGNELUTTI FILHO, A.; EVANGELISTA, D.H.R.; GONÇALVES, E.C.P.; STORCK, L. Tamanho de amostra de caracteres de genótipos de soja. Ciência Rural, Santa Maria, v.39, n.4, p.983-991, 2009.

CARGNELUTTI FILHO, A.; LOPES, S.J.; BRUM, B.; SILVEIRA, T.R.; TOEBE, M.; STORCK, L. Tamanho de amostra de caracteres em híbridos de mamoneira. Ciência Rural, Santa Maria, v.40, n.2, p.250-257, 2010a.

CARGNELUTTI FILHO, A.; TOEBE, M.; SILVEIRA, T.R.; CASAROTTO, G.; HAESBAERT, F.M.; LOPES, S.J. Tamanho de amostra e relações lineares de caracteres morfológicos e produtivos de crambe. Ciência Rural, Santa Maria, v.40, n.11, p.2262-2267, $2010 b$.

CATAPATTI, T.R.; GONÇALVES, M.C.; SILVA NETO, M.R.; SOBROZA, R. Tamanho de amostra e número de repetições para avaliação de caracteres agronômicos em milho-pipoca. Ciência e Agrotecnologia, Lavras, v.32, n.3, p.855-862, 2008.

COELHO, R.I.; LOPES, J.C.; CARVALHO, A.J.C.; AMARAL, J.A.T.; MATTA, F.P. Estado nutricional e características de crescimento do abacaxizeiro jupi cultivado em latossolo amarelo distrófico em função da adubação com NPK. Ciência e Agrotecnologia, Lavras, v.31, n.6, p.1696-1701, 2007. 
CUNHA, G.A.P. da; CABRAL, J.R.S.; SOUZA, L.F. da S. Abacaxizeiro: cultivo, agroindústria e economia. Brasília: Embrapa, 1999. 480p.

FERNANDES, E.N.; SILVA, P.S.L. Tamanho da amostra e método de amostragem para caracteres da espiga do milho. Ciência e Agrotecnologia, Lavras, v.20, n.2, p.252-256, 1996.

HAESBAERT, F.M.; SANTOS, D.; LÚCIO, A.D.; BENZ, V.; ANTONELLO, B.I.; RIBEIRO, A.L.P. Tamanho de amostra para experimentos com feijão-de-vagem em diferentes ambientes. Ciência Rural, Santa Maria, v.41, n.1, p. 38-44, 2011.

LEITE, M.S.O.; PETERNELLI, L.A.; BARBOSA, M.H.P.; CECON, P.R.; CRUZ, C.D. Sample size for full-sib family evaluation in sugarcane. Pesquisa Agropecuária Brasileira, Brasília, v.44, n.12, p.1562-1574, 2009.

LÚCIO, A.D.; VIEIRA NETO, J.; CHIARADIA, L.A.; STORCK, L. Distribuição espacial e tamanho de amostra para o ácaro do bronzeado da erva-mate. Revista Árvore, Viçosa, v.33, n.1, p.145-150, 2009.

PAULA, M.B.; CARVALHO, V.D.; NOGUEIRA, F.D.; SOUZA, L.F.S. Efeito da calagem, potássio e nitrogênio na produção e qualidade do fruto do abacaxizeiro. Pesquisa Agropecuária Brasileira, Brasília, v.26, n.9, p.1337-1343, 1991.

PAULA, M.B.; HOLANDA, F.S.R.; MESQUITA, H.A.; CARVALHO, V.D. Uso da vinhaça no abacaxizeiro em solo de baixo potencial de produção. Pesquisa Agropecuária Brasileira, Brasília, v.34, n.7, p.1217-1222, 1999.

PAULA, M.B.; MESQUITA, H.A.; NOGUEIRA, F.D. Nutrição e adubação do abacaxizeiro. Informe Agropecuário, Belo Horizonte, v.19, p.33-39, 1998.
SAEG. Sistema para análises estatísticas. Versão 9.1: Viçosa: UFV, Fundação Arthur Bernardes, 2007.

SILVA, A.P.; ALVAREZ, V.H.; SOUZA, A.P.; NEVES, J.C.L.; NOVAIS, R.F.; DANTAS, J.P. Sistema de recomendação de fertilizantes e corretivos para a cultura do abacaxi - fertcalc-abacaxi. Revista Brasileira de Ciência do Solo, Viçosa, v.33, n.5, p.1269-1280, 2009.

SILVA, J.; SILVA, P.S.L.; NUNES, R.P. Amostragem e tamanho da amostra na estimação de caracteres da espiga do milho. Pesquisa Agropecuária Brasileira, Brasília, v.33, n.12, p.1983-1988, 1998.

SOUZA, O.P.; TEODORO, R.E.F.; MELO, B.; TORRES, J.L.R. Qualidade do fruto e produtividade do abacaxizeiro em diferentes densidades de plantio e lâminas de irrigação. Pesquisa Agropecuária Brasileira, Brasília, v.44, n.5, p.471-477, 2009.

SPIRONELLO, A.; QUAGGIO, J.A.; TEIXEIRA, L.A.J.; FURLANI, P.R.; SIGRIST, J.M.M. Pineapple yield and fruit quality effected by NPK fertilization in a tropical soil. Revista Brasileira de Fruticultura, Jaboticabal, v.26, n.2, p.155-159, 2004.

STORCK, L.; LOPES, S.J.; CARGNELUTTI FILHO, A.; MARTINI, L.F.D.; CARVALHO, M.P. Sample size for single, double and triple hybrid corn ear traits. Scientia Agrícola, Piracicaba, v.64, n.1, p.30-35, 2007.

VELOSO, C.A.C.; OEIRAS, A.H.L.; CARVALHO, E.J.M.; SOUZA, F.R.S. Resposta do abacaxizeiro à adição de nitrogênio, potássio e calcário em latossolo amarelo do nordeste Paraense. Revista Brasileira de Fruticultura, Jaboticabal, v.23, n.2, p.396-402, 2001. 